\title{
Perfil Social e Funcional dos Usuários da Estratégia Saúde da Família com Acidente Vascular Encefálico
}

\author{
Social and Functional Profile of Userswith Stroke Assisted \\ by the Family Health Strategy
}

\author{
CANDICE SIMÕES PIMENTA DE MEDEIROS ${ }^{1}$ \\ OZAIRARGENTILLE PEREIRADA SILVA ${ }^{1}$ \\ JUSCIELE BEZERRADE ARAÚJO' \\ DAMIÃO ERNANE DE SOUZA ${ }^{2}$ \\ ENIO WALKER AZEVEDO $\mathrm{CACHO}^{3}$ \\ ROBERTA DE OLIVEIRA CACHO ${ }^{4}$
}

\section{RESUMO}

Objetivo: Analisar o perfil social e funcional dos usuários de Unidade Básica de Saúde/Estratégia Saúde da Família com Acidente Vascular Encefálico (AVE) em Santa Cruz, município do estado do Rio Grande do Norte. Material e Métodos: Tratase de um estudo transversal constituído por 39 indivíduos com diagnóstico clínico de AVE. Foram avaliados o perfil sociodemográfico e o comprometimento cognitivo, funcional e sensório motor através das escalas: Mini Exame do Estado Mental (MEEM), Medida de Independência Funcional (MIF), Escala de Equilíbrio de Berg (EEB), Protocolo de Desempenho Físico Fugl-Meyer (FM), Timed Up and Go (TUG) e a escala de Deambulação Funcional (FAC). Resultados: A idade média dos indivíduos foi $68,94 \pm 14,01$ anos onde $56,41 \%$ eram mulheres, $58,97 \%$ casados, $58,97 \%$ pardos, $46,15 \%$ analfabetos, $41,03 \%$ agricultores, $71,79 \%$ aposentados $\mathrm{e}$ $84,62 \%$ residentes em casa própria. 0 hemisfério cerebral esquerdo $(56,41 \%)$ e o tipo isquêmico $(61,54 \%)$ mostraram-se mais prevalentes nesta população. $O$ tempo de lesão após o AVE foi de $6,7 \pm 6,9$ anos, onde $66,67 \%$ apresentaram um único evento. $97,40 \%$ dos avaliados apresentavam fatores de risco associados. A Hipertensão Arterial Sistêmica (HAS) $(74,35 \%)$ foi o de maior destaque. No MEEM observou-se $82,05 \%$ da amostra com grave comprometimento cognitivo, $48,72 \%$ com pontuação da MIF indicando maior independência funcional, grave comprometimento motorna FM $(46,88 \%)$ e equilíbrio pobre com significativo risco para quedas na pontuação da EEB (43,59\%) e TUG (36\%). Conclusão: O elevado acometimento do AVE está relacionado com as características epidemiológicas da região,assim como os déficits funcionais, cognitivos e sensório motores encontrados evidenciando um grave problema de saúde pública.

DESCRITORES

Acidente Vascular Cerebral. Epidemiologia. Saúde Coletiva.

\begin{abstract}
Objective: To analyze the social and functional profile of users with stroke assisted by the Primary Care Health Unit/ Family Health Strategy in the city of Santa Cruz, Rio Grande do Norte State, Brazil. Materials and Methods: This was a cross-sectional study including 39 individuals with a clinical diagnosis of stroke. The sociodemographic, cognitive, functional and sensory motor impairment profiles of the users were evaluated using the following tools: Mini-Mental State Examination (MEEM), Functional Independence Measure (MIF), Berg Balance Scale (EEB), Fugl-Meyer Assessment Scale (FM), Timed Up and Go (TUG) and Functional Ambulation Scale (FAC). Results: The mean age of individuals was $68.94 \pm 14.01$ years, where $56.41 \%$ were woman, $58.97 \%$ married, $58.97 \%$ mixed race, $46.15 \%$ illiterate, $41.03 \%$ farmers, $71.79 \%$ retirees, and $84.62 \%$ living in their own house. The leftcerebral hemisphere $(56.41 \%)$ was the most affected side and ischemic stroke $(61.54 \%)$ the most prevalent condition in this population. The injury time after stroke was $6.7 \pm 6.9$ years, and $66.67 \%$ of the individual shad a single stroke; $97.4 \%$ of them presented as sociated risk factors, particularly systemic arterial hypertension (HAS) $(74.35 \%)$. The MEEM indicated that $82.05 \%$ of the sample had severe cognitive impairment; $48.72 \%$ of the sample showed MIF score indicative of functional independence; $46.88 \%$ showed severe motor impairment in $\mathrm{FM}$; and poor balance with significant risk for falls was found to be $43.59 \%$ and $36 \%$ based on EEB and TUG scores, respectively. Conclusion: The high prevalence of stroke is related with the epidemiological characteristics of the region as well as with functional, cognitive and sensory motor deficits, which indicates a serious public health problem.
\end{abstract}

\section{DESCRIPTORS}

Stroke. Epidemiology. Public Health.

Fisioterapeuta. Santa Cruz. Rio Grande do Norte. Brasil.

Analista de Gestão e Pesquisa do IBGE/RN. Natal. Rio Grande do Norte. Brasil.

3 Docente Doutor do Curso de Fisioterapia da Faculdade de Ciências da Saúde do Trairi (Facisa/UFRN). Santa Cruz. Rio Grande do Norte. Brasil. 4 Docente Doutora do Curso de Fisioterapia da Faculdade de Ciências da Saúde do Trairi (Facisa/UFRN). Santa Cruz. Rio Grande do Norte. Brasil. 
$\mathrm{O}$ Acidente Vascular Encefálico (AVE) corresponde a uma síndrome neurológicaaguda, de origem vascular com início rápido e súbito, causada por uma interrupção do fluxo sanguíneo para áreas focais do encéfalo, podendo ocorrer na sua forma isquêmica ou hemorrágica ${ }^{1}$. O AVE isquêmico é decorrente da insuficiência de suprimento sanguíneo cerebral ocasionado principalmente por placas ateroscleróticas; já o evento hemorrágico tem a hemorragia hipertensiva como causa mais comum de lesão, seguida dos aneurismas saculares e malformações arteriovenosas (MAV's) ${ }^{2}$. Suas manifestações clínicas e prognóstico vão depender da localização e extensão da lesão ${ }^{3}$. Por se tratar de uma das principais causas de morbi-mortalidade no mundo, além de proporcionar incapacidades motoras e cognitivas, o AVE é uma doença comum e de grande impacto na saúde pública ${ }^{2-4}$.

No Brasil, o AVE ainda é uma das principais causas de morte ${ }^{4,6}$, onde as projeções para o ano de 2015 e 2030 expressam números alarmantes, com expectativa de 18 e 23 milhões de novos casos no mundo 5 . De acordo com o DATASUS, o estado do Rio Grande do Norte apresentou coeficiente de mortalidade por doenças cerebrovasculares no anode 2008, com valorde 43,5/100 mil habitantes. O município de Santa Cruz - RN apresentou no ano de 2011 a taxa de internações por AVE de 8,65/10.000 habitantes e o coeficiente de mortalidade por doenças cerebrovasculares no ano de 2008 de 66,2/100 mil habitantes ${ }^{7}$.

As crescentes mudanças no estilo e expectativa de vida associadas com o aumento dos fatores de risco para as doenças cerebrovasculares podem servir como justificativa para a grande incidência do $\mathrm{AVE}^{8,9}$. O conhecimento e percepção dos fatores de risco são medidas essenciais para se obter um maior controle da doença e melhor adesão a medidas preventivas.Dentre os principais fatores de risco que podem aumentar a propensão ao AVE, destacam-se os altos perfis lipídicos, diabetes mellitus, sobrepeso e obesidade, além do tabagismo e sedentarismo ${ }^{10}$. Um estudo realizado em Fortaleza-CE mostrou que $77,2 \%$ dos pacientes com AVE eram hipertensos, $23,5 \%$ diabéticos, 3,9\% tabagistas e $21,2 \%$ etilistas ${ }^{8}$. Além disso, os principais dados sobre epidemiologia e fatores de risco associados ao AVE são baseados principalmente em estudos norte-americanos ou europeus e existe uma escassez de resultados dessa patologia em países em desenvolvimento.
O Programa Saúde da Família (PSF) foiimplantado no Brasil em 1994, como uma importante estratégia de reorientação do modelo assistencial a partir da atenção básica, em conformidade com os princípios do Sistema Único de Saúde e assistência voltado para a comunidade 2,11 .A Estratégia Saúde da Família (ESF)visa à reorganização da atenção básica favorecendo a reorientação do processo de trabalho,ampliando a resolutividade e impacto na situação de saúde das pessoas e coletividades. A ESF corresponde a um ambiente onde devem ser priorizadas estratégias de prevenção, promoção e proteção da saúdee identificação precoce e o tratamento das doenças, onde o AVE deve ser pauta das ações haja vista as suas limitações e impacto social além do crescente número de fatores de risco para doença adscritos na população ${ }^{6}$.

As repercussões e sequelas do AVE propiciam diversas alterações pessoais e sociais na vida dos indivíduos, que por sua vez geram restrições e alterações relacionadas a limitações nas Atividades de Vida Diárias (AVD's). A funcionalidade e motricidade ficam completamente afetados e as incapacidades e sequelas geradas acarretam limitações funcionais e grande dependência ${ }^{12}$. A utilização de instrumentos de avaliação para pacientes pós-AVE é de extrema importância para analisar e compreender o impacto das limitações dessa patologia além de sua correlação com as alterações do comprometimento sensório-motor, sensitivo e funcional. Diversas escalas de avaliação para o AVE são usadas na prá-tica clínica e em pesquisas para diagnósticos, prognósticos, resposta a tratamentos e avaliação da severidade clínica ${ }^{12,13}$.

Os estudos sobre a epidemiologia por doenças cerebrovasculares são necessários para que se conheça sua distribuição por localidade, a fim de subsidiar políticas de atenção aos agravos agudos e crônicos mais prevalentes. Além disso, mesmo com a alta taxa de morbi-mortalidade em países menos desenvolvidos, ainda existem poucas informações sobre a prevalência de doenças neurológicas, dentre elas o AVE.As pesquisas e publicações com levantamento epidemiológico e caracterização funcional e sensório motora dos indivíduos acometidos por AVE inseridos na ESF são bastante escassos e esses resultados são primordiais para que se possa aumentar a visibilidade desse problema. Dessa forma, o presente estudo teve como objetivo analisar o perfil social e funcional dos usuários de Unidades Básicas 
de Saúde (UBS)/Estratégia Saúde da Família (ESF) com Acidente Vascular Encefálico (AVE)acima de 50 anos do município de Santa Cruz - Rio Grande do Norte.

\section{MATERIAL E MÉTODOS}

Trata-se de um estudo transversal e descritivo, realizado no período de julho de 2011 a dezembro de 2012 onde foram selecionados e investigados os sujeitos com diagnóstico clínico de AVE adscritos em todas as Unidades Básicas de Saúde (UBS) da cidade de Santa Cruz-RN. A cidade de Santa Cruz está localizada a $122 \mathrm{~km}$ da capital do estado, Natal, na microrregião da Borborema Potiguar no Rio Grande do Norte com estrutura territorial $624.356 \mathrm{~km}^{2}$, área de densidade demográfica de $57,33 \mathrm{hab} / \mathrm{km}^{2}$, apresentando uma população estimada de 38.924 habitantes ${ }^{14}$. Além disso, a cidade contempla cerca de 17.472 habitantes homens e 18.325 habitantes mulheres ${ }^{14}$, onde todas as UBS funcionam sob a lógica ESF visando a integralidade ao cuidado em saúde.

Foram incluídos na pesquisa os indivíduos com idade superior a 50 anos com diagnóstico clínico de AVE apresentando ou não sinais clínicos de sequela. Os sujeitos com qualquer outra patologia neurológica associada foram excluídos da pesquisa assim como os casos de desistência e mudança de cidade.

Após contato com as UBS dos bairros Centro, Conjunto Cônego Monte, Paraíso, Maracujá e DNER, os entrevistadores realizaram coleta de dados em prontuários e agendaram visita domiciliar para entrevista e análise das condições clínicas do paciente. A entrevista foi realizada por dois avaliadores que foram devidamente capacitados para aplicar todos os instrumentos de avaliação.

A caracterização sociodemográfica se deu através de questionário semi-estruturado onde foram preenchidas informações sobre nome, sexo, idade, escolaridade, estado civil, ocupação, fonte de renda, tempo decorrido desde o primeiro AVE, hemicorpo acometido. Além, da aplicação das escalas de avaliação: Mini-Exame do Estado Mental ${ }^{15}$, Medida de Independência Funcional ${ }^{16}$, Escala de Equilíbrio de Berg ${ }^{17}$, Escala de Desempenho Físico de FuglMeyer ${ }^{18}$, Escala de Categorias de Deambulação Funcional ${ }^{19} \mathrm{e}$ Timed Up and $\mathrm{Go}^{20}$.

Para mensurar o comprometimento cognitivo foi utilizado o Mini Exame do Estado Mental (MEEM). O MEEM é dividido em sete dimensões, que incluem orientação temporal, orientação espacial, memória imediata, atenção e cálculo, evocação, linguagem e construção visual. A sua pontuação total do MEEM varia de 0 a 30 pontos, onde alguns autores estabeleceram o ponto de corte para os analfabetos (13 pontos), de 1 a 8 anos de estudo (18 pontos) e mais de 8 anos de escolaridade (26 pontos). A pontuação para os alfabetizados vai de 30 a 26 (função cognitiva preservada), 26 a 24 (alteração não sugestiva de déficit) e menor, igual a 23 (déficits cognitivos). Este instrumento foi validado no Brasil, sendo levado em consideração à idade e o nível de escolaridade dos indivíduos participantes da pesquisa ${ }^{15}$.

A Medida de Independência Funcional (MIF) foi utilizada para medir o nível de independência funcional dos sujeitos e compreende um conjunto de 18 tarefas, referentes às subescalas de autocuidado, controle esfincteriano, transferências, locomoção, comunicação e cognição social. Cada item pode ser classificado em graus de dependência de 7 níveis, sendo o valor 1 correspondente à dependência total e o valor de 7 corresponde à normalidade na realização de tarefas de forma independente; assim a pontuação total varia de 18 a $126^{16}$

O equilíbrio foi avaliado pela Escala de Equilíbrio de Berg (EEB). A escala compreende 14 tarefas onde cada uma recebe uma pontuação de 0 a 4 (0, incapaz de realizar; 4, completa a tarefa) que classificam a qualidade do desempenho da tarefa, o tempo gasto para concluir a tarefa ou para manter-se em uma determinada postura. Somadas todas as atividades, obtém-se uma pontuação máxima de 56 pontos, sendo que de 0 a 20 são considerados indivíduos com equilíbrio pobre e de 40 a 56 , com um bom equilíbrio ${ }^{17}$.

O comprometimento motor e sensitivo foi avaliado pela Escala de Desempenho Físico de FuglMeyer (FM). Esta escala possui um total de 226 pontos que são divididos em 5 sub-itens: função motora (100 pontos no total, sendo 66 para membro superior e 34 para membro inferior), função sensorial (24 pontos), equilíbrio (14 pontos), amplitude de movimento (44 pontos) e dor articular (44 pontos). Cada um destes itens são pontuados por uma escala ordinal de 3 pontos ( 0 - não pode realizar, 1 - realiza parcialmente, 2 - realiza completamente). A função motora sugere a severidade do AVE: $0-$ 35 , comprometimento motor severo; 36-55, comprometimento motor severamente moderado; 56-79, comprometimento motor moderado; > 80, comprometimento motor leve ${ }^{18}$. 
A escala de Categorias de Deambulação Funcional (Functional Ambulatory Cathegory - FAC) classifica a capacidade do paciente em deambular e é dividida em seis níveis: 0 , o paciente não pode andar ou requer auxílio de duas ou mais pessoas; 1, O indivíduo precisa de suporte contínuo de uma pessoa que suporte seu peso e ajude com seus passos, equilíbrio e coordenação; 2, O indivíduo precisa de leve suporte (contínuo ou intermitente) de uma pessoa que ajude com o equilíbrio e coordenação; 3 , O indivíduo deambula em superfície plana sem contato manual mas precisa de uma pessoa para supervisão ou dicas verbais; 4,0 indivíduo pode deambular independente em superfície plana mas requer supervisão para escadas, rampas e superfícies irregulares; 5,0 indivíduo pode andar independente em qualquer lugar, incluindo escadas ${ }^{19}$.

A fim de analisar o equilíbrio dinâmico em conjunto com a velocidade da marcha utilizou-se o Timed Up and Go - TUG. O teste é realizado a partir de uma posição inicial sentada, com as costas apoiadas em uma cadeira, sem o auxílio dos membros superiores, e solicitado a andar um percurso linear de 3 metros até um ponto prédeterminado por uma marca no chão, girar $180^{\circ}$, retornar e sentar novamente, apoiando as costas na mesma cadeira. O paciente é instruído a realizar o teste usando seus calçados habituais, a não conversar durante a execução e a realizá-lo em velocidade normal, de forma segura. São verificados o número de passos dados durante o percurso (para cálculo de cadência: passos/minuto) e o tempo gasto na realização da tarefa (em segundos). Para tempo inferior a 20 segundos, o paciente apresenta baixo risco de quedas; entre 20 e 29 segundos, médio risco de quedas e acima de 30 segundos, alto risco de quedas ${ }^{20}$.

O estudo foi aprovado pelo Comitê de Ética da UFRN (\#060/2011) e os sujeitos que concordaram em participar do estudo assinaram um Termo de Consentimento Livre e Esclarecido.

Os dados coletados foram tabulados no software Microsoft Office Excel e posteriormente analisados de forma descritiva através do BioEstat 5.0. Foram calculados os valores de frequência absoluta ( $n$ ) e percentual (\%) das variáveis categóricas, a média e o desvio padrão das variáveis contínuas.

\section{RESULTADOS}

Foram encontrados e notificados 97 casos de AVE em todas as UBS/ESF de Santa Cruz/RN, porém, somente 39 indivíduos preencheram os critérios de inclusão. Dos 58 excluídos, 15 se enquadraram nos critérios de exclusão, dois desistiram de participar do estudo e 41 foram desvinculados da pesquisa, poismudaram de cidade.

A distribuição dos indivíduos com AVE segundo os fatores sociodemográficos está descrita na Tabela 1. Os resultados encontrados apontam para predominância feminina $(56,41 \%)$, sendo o estado civil mais prevalente o casado (58,97\%). A média de idade dos indivíduos foi de 68,94 anos $( \pm 14,01)$. O nível de escolaridade predominante foi o de analfabetos $(46,15 \%)$. A raça foi autoreferida e $58,97 \%$ denominou-se como pardos. A agricultura representou a principal fonte de ocupação $(41,03 \%)$ e como era de se esperar, para a faixa etária encontrada, cerca de $71,79 \%$ eram aposentados e residentes de casa própria $(84,62 \%)$ (Tabela 1$)$.

Com relação às características clínicas, o AVE no hemisfério esquerdo (56,41\%) e a etiologia do tipo isquêmica $(61,54 \%)$ foram predominantes. Cerca de $97,4 \%$ dos avaliados apresentavam fatores de risco associados, onde a Hipertensão Arterial Sistêmica (HAS) equivaleu a $74,35 \%$ da amostra. A caracterização dos pacientes de acordo com os aspectos clínicos encontra-se descrita na Tabela 2.

Em relação à aplicação dos instrumentos de avaliação, observou-se que o teste do Mini Exame do Estado Mental (MEEN) obteve uma pontuação média de $17,1 \pm 7,4$ pontos, onde 32 pacientes $(82,05 \%)$ foram classificados com um grave comprometimento e déficits cognitivos. $\mathrm{Na}$ Medida de Independência Funcional (MIF) obtevese uma média de $80,4 \pm 34,4$ pontos, onde 19 pacientes $(48,72 \%)$ apresentaram independência funcional. A Escala de Equilíbrio de Berg (EEB) obteve média de $24,5 \pm 18,5$, onde 17 pacientes $(43,59 \%)$ obtiveram equilíbrio pobre. Na Escala de Desempenho Físico de Fugl-Meyer (FM), somente 32 pacientes completaram a avaliação com a pontuação média total de $48,3 \pm 32,3$. Os outros 6 pacientes não completaram a avaliação pois o lado afetado não foi identificado ou a hemiplegia era bilateral. Dos avaliados, 15 (46,88\%) apresentaram um grave grau de comprometimento motor. $\mathrm{Na}$ aplicação do teste Time Up And Go (TUG) 14 pacientes não conseguiram realizar o teste. Dentre os 25 pacientes avaliados, obteve-se a média, em 


\begin{tabular}{|c|c|c|c|}
\hline \multicolumn{4}{|c|}{$\begin{array}{l}\text { Tabela 1. Caracterização dos indivíduos com AVE de acordo com os aspectos } \\
\text { sociodemográficos no período de junho de } 2011 \text { a dezembro de 2012. Santa Cruz - } \\
\text { Rio Grande do Norte, Brasil. }\end{array}$} \\
\hline \multicolumn{2}{|r|}{ Variáveis } & $\mathbf{N}$ & $\%$ \\
\hline \multirow[t]{2}{*}{ Sexo } & Feminino & 22 & 56,41 \\
\hline & Masculino & 17 & 43,59 \\
\hline Idade (Anos) & \multicolumn{3}{|c|}{$68,94 \pm 14,01$} \\
\hline \multirow{3}{*}{ Estado Civil } & Casado & 23 & 58.97 \\
\hline & Viúvo & 11 & 28,21 \\
\hline & Solteiro & 5 & 12,82 \\
\hline \multirow{4}{*}{ Escolaridade } & Analfabetos & 18 & 46.15 \\
\hline & Primeira à quarta série & 8 & 20,51 \\
\hline & Alfabetizados & 6 & 15,38 \\
\hline & $\begin{array}{l}\text { Segundo grau ou ensino } \\
\text { superior completo ou } \\
\text { incompleto }\end{array}$ & 7 & 17,95 \\
\hline \multirow{4}{*}{ Etnia } & Pardos & 23 & 58.97 \\
\hline & Brancos & 10 & 25,64 \\
\hline & Neqros & 5 & 12,82 \\
\hline & Amarelos & 1 & 2,56 \\
\hline \multirow{3}{*}{ Ocupação } & Aaricultura & 16 & 41,03 \\
\hline & Atividades do lar & 5 & 12,82 \\
\hline & Outros & 18 & 46.15 \\
\hline \multirow{3}{*}{$\begin{array}{l}\text { Fonte de } \\
\text { Renda }\end{array}$} & Aposentadoria & 28 & 71,79 \\
\hline & Pensão & 5 & 12,82 \\
\hline & $\begin{array}{l}\text { Pensão e aposentadoria, } \\
\text { ajuda de terceiros }\end{array}$ & 6 & 15,38 \\
\hline \multirow{2}{*}{$\begin{array}{l}\text { Tipo de } \\
\text { Moradia }\end{array}$} & Casa Própria & 33 & 84,62 \\
\hline & Casa Alugada & 6 & 15,38 \\
\hline
\end{tabular}

\begin{tabular}{|c|c|c|c|}
\hline \multirow{5}{*}{$\begin{array}{c}\text { Hemisfério Cerebral } \\
\text { Acometido }\end{array}$} & & $\mathbf{N}$ & $\%$ \\
\hline & Esquerdo & 22 & 56,41 \\
\hline & Direito & 10 & 25.64 \\
\hline & Direito e Esquerdo & 3 & 7,69 \\
\hline & Não definido & 4 & 10.26 \\
\hline \multirow{3}{*}{ Etiologia do AVE } & Isquêmico & 24 & 61.54 \\
\hline & Hemorráaico & 9 & 23.08 \\
\hline & Sem identificação & 6 & 15,38 \\
\hline $\begin{array}{c}\text { Tempo de } \\
\text { Lesão(Anos) }\end{array}$ & \multicolumn{3}{|c|}{$6,7 \pm 6,9$} \\
\hline \multirow{3}{*}{ Eventos de AVE } & Único evento & 26 & 66,67 \\
\hline & Dois eventos & 9 & 23,08 \\
\hline & Três eventos & 4 & 10,26 \\
\hline \multirow[b]{2}{*}{ Presença de FR } & Sim & 38 & 97,4 \\
\hline & Não & 1 & 2,6 \\
\hline \multirow{8}{*}{ FR } & HAS & 29 & 74,35 \\
\hline & Sedentarismo & 23 & 58,97 \\
\hline & DM & 18 & 46,15 \\
\hline & Doenças Cardíacas & 17 & 43,58 \\
\hline & Tabagismo & 12 & 30,76 \\
\hline & Colesterol Elevado & 7 & 17,94 \\
\hline & Etilismo & 6 & 15,38 \\
\hline & Obesidade & 3 & 7,69 \\
\hline
\end{tabular}

Legenda: FR, Fatores de risco; HAS, Hipertensão Arterial Sistêmica; DM, Diabetes Mellitus. Período: iulho de 2011 adezembro de 2012. 
segundos, de $29,7 \pm 27,5$ onde 13 indivíduos (52\%) foram classificados com baixo risco de quedas. $\mathrm{Na}$ aplicação da Escala de Deambulação Funcional (FAC), cerca de $30,77 \%$ dos avaliados apresentaram marcha independente (Tabela 3).

\section{DISCUSSÃO}

Os resultados do presente estudo apontam o acometimento do AVE predominante no sexo feminino, com idade média de 68 anos, casados e

\begin{tabular}{|c|c|c|c|c|}
\hline ESCALA & CLASSIFICACÃO & $\mathbf{n}$ & $\%$ & Média Total \\
\hline \multirow{3}{*}{ MEEM (n =39) } & Déficit coanitivo & 32 & 82,05 & \\
\hline & Sem déficit & 5 & 12,82 & $17,1( \pm 7,4)$ \\
\hline & Coanição preservada & 2 & 5,13 & \\
\hline \multirow{3}{*}{ MIF $(n=39)$} & Grave & 5 & 12,82 & \\
\hline & Moderado & 15 & 38,4 & $80,4( \pm 34,4)$ \\
\hline & Independência funcional & 19 & 48,72 & \\
\hline \multirow{3}{*}{ EEB $(n=39)$} & Equilíbrio pobre & 17 & 43,59 & \\
\hline & Equilíbrio moderado & 11 & 28,21 & $24,5( \pm 18,5)$ \\
\hline & Equilíbrio bom & 11 & 28.21 & \\
\hline \multirow{4}{*}{ FM $(n=32)$} & Comprometimento arave & 15 & 46,88 & \\
\hline & Comprometimento qrave-moderado & 12 & 37.50 & $48,3( \pm 32,3)$ \\
\hline & Comprometimento moderado & 4 & 12,5 & \\
\hline & Comprometimento leve & 1 & 3,13 & \\
\hline \multirow{3}{*}{ TUG (n=25) } & Alto risco de quedas & 9 & 36 & \\
\hline & Baixo risco de quedas & 13 & 52 & $29,7( \pm 27,5)$ \\
\hline & Risco normal de quedas & 3 & 12 & \\
\hline \multirow{5}{*}{$\mathrm{FAC}(n=39)$} & Não pode andar & 9 & 23,07 & \\
\hline & Necessidade de suporte contínuo & 5 & 12,82 & \\
\hline & Auxilio no equilíbrio e coordenação & 4 & 10,26 & - \\
\hline & $\begin{array}{c}\text { Ajuda com escadas e superfícies } \\
\text { irrequlares }\end{array}$ & 9 & 23,08 & \\
\hline & Marcha independente & 12 & 30,77 & \\
\hline
\end{tabular}

Legenda: MEEM, Mini Exame do Estado Mental; MIF, Medida de Independência Funcional; EEB, Escala de Equilíbrio de Berg; FM, Escala de Desempenho Físico Fugl-Meyer; TUG, Timed Up and Go; FAC, Escala de Deambulação Funcional. Período: Julho de 2011 à Dezembro de 2012.

de raça parda com a presença de uma amostra heterogênea. O predomínio do AVE no sexo feminino corrobora com alguns achados da literatura ${ }^{5,21}$. As mulheres apresentam fatores potenciais que aumentam a chance de desenvolver a doença, como altos níveis de glicemia, o uso de contraceptivos orais, enxaquecas, HAS, DM, dislipidemia e doenças tromboembólicas ${ }^{28}$. Além disso, o predomínio no sexo feminino também pode ser atribuído à faixa etária, haja vista que as mulheres apresentam maior expectativa de vida.

Em um estudo com 180 pacientes com AVC em Fortaleza - CE, foi observado que 53,9\% da amostra era composta por indivíduos com companheiros e com idade média de 62,3 anos $^{8}$. Em Natal - RN, em um estudo descritivo com hemiplégicos pós-AVC, observaram a idade média de 65,9 anos e $50 \%$ da amostra composta por pacientes casados ${ }^{1}$ corroborando com os resultados encontrados no presente estudo. Em um estudo no Paraná, observou-se que $76,9 \%$ dos óbitos ocorreram na faixa etária igual ou superior a 65 anos $^{22}$. O aumento progressivo do acometimento do AVE de acordo com a idade é esperado, uma vez que a idade é o principal fator de risco não modificável, reforçando ainda a necessidade de prevenção e controle de fatores de risco para doença cerebrovascular ${ }^{4}$.

No presente estudo, observou-se predominância de indivíduos analfabetos, residentes em casa própria e que a principal fonte de renda era a aposentadoria. Cavalcante et al., encontraram em sua amostra de pacientes com AVE, uma escolaridade média de 4,3 anos e renda de 513 reais $^{8}$. Enquanto isso, no município de Vassouras - RJ, $61 \%$ da amostra com AVC avaliada era 
composta por aposentados, $70 \%$ residentes em zona urbana, $46 \%$ com grau de escolaridade até a $4^{\circ}$ série e $26 \%$ de analfabetos ${ }^{5}$. Dessa forma, observa-se no presente estudo uma alta porcentagem de indivíduos com baixos níveis de escolaridade, chamando atenção para o número de analfabetos que reflete nas condições intrínsecas da população do município onde, de acordo com o Censo demográfico do IBGE (2010) ${ }^{14}, 4.797$ pessoas correspondem a uma parcela da população residente que nunca frequentou creche ou escola na faixa etária até 60 anos ou mais. Essa característica também reflete na incidência do AVE na população, já que a baixa quantidade de informações sobre os meios de prevenção, os hábitos e comportamentos de risco à saúde fazem com que o segmento populacional de baixa escolaridade esteja mais suscetível ao AVE.

A etnia parda foi a mais referida no estudo, entretanto esse resultado não corrobora com os dados encontrados na literatura onde a etnia negra é mais descrita como maior prevalência para o $\mathrm{AVE}^{4}$. Essa divergência encontrada pode ser justificada pelos diferentes modos de avaliação da etnia, já que no estudo em questão utilizou-se a avaliação auto-referida. A investigação das características socioeconômicas, como a etnia, nível educacional e renda permitem observar que a condição social associada com a falta de informações, sobre prevenção e fatores de risco à saúde deixam os indivíduos mais propensos a um evento de AVE.

$O$ acometimento do AVE foi maior no hemisfério cerebral esquerdo, predominando a partir da forma isquêmica. Os achados encontrados na literatura remetem a um maior acometimento do AVE isquêmico. Estudos como Pereira et al., encontraram $62,2 \%$ de casos isquêmicos ${ }^{4}$ e Costa et al., observaram $90 \%$ de casos isquêmicos ${ }^{1}$. A maior abrangência do AVE isquêmico encontrada no estudo está intimamente associada e correlacionada com a prevalência dos principais fatores de risco. Desse modo, o impacto da lesão isquêmica, sua gravidade, localização e extensão são extremamente relevantes para o quadro de morbi-mortalidade.

Em relação ao tempo de lesão houve uma média de 6,7 anos,onde $97,40 \%$ dos indivíduos avaliados tinham algum tipo de fator de risco para o AVE. Diversos estudos relacionados aos principais fatores de risco para o AVE corroboram com os resultados encontrados no presente estudo. Segundo Rosamond et al., os principais fatores de risco que podem aumentar a propensão ao AVE são os altos perfis lipídicos, diabetes mellitus, sobrepeso e obesidade, além do tabagismo e sedentarismo ${ }^{10}$. Os estudos mostram a elevada incidência da HAS bem como sua significativa potencialidade para ocasionar o AVE ${ }^{6,16}$. A HAS é considerada como um dos principais fatores de risco modificáveis para o AVE e um dos mais importantes problemas de saúde pública, gerando maior propensão para o desenvolvimento de doenças cerebrovasculares ${ }^{23}$. Sendo o principal fator de risco para o acometimento do AVE encontrado no estudo, essa alta prevalência pode ser justificada já que a idade elevada é um dos fatores para a presença da HAS.A DM aparece como o segundo principal fator de risco do estudo e é um elemento determinante representando um grave risco para o desenvolvimento do AVE, gerando alterações nos vasos, desordem hemodinâmica e formação de placas ateroscleróticas. Dessa forma, como o AVE é um processo patológico de origem multifatorial, a ação de vários fatores de risco pode ser o elo para ocasionar as mais diversas comorbidades e sequelas.

Ainda se sabe pouco sobre como, o tempo e em que extensão as modificações nos principais fatores de risco podem refletir de forma significativa na mortalidade do $\mathrm{AVE}^{24}$. Porém, a identificação e o controle dos fatores de risco são medidas essenciais e prioritárias para a prevenção primária na população, pois permitem uma redução significativa da incidência e recidiva do AVC, por meio de mudança nos hábitos de vida, além de ser uma obrigação para os envolvidos com os cuidados em saúde. Caracterizar determinantes de recorrência e mortalidade após o AVE reflete a prevenção secundária, haja vista que a recorrência do AVE ainda é a maior ameaça para os sobreviventes ${ }^{21,24,18}$. As consequências, em longo prazo, após o AVE vão depender do tamanho e localização da lesão, além da capacidade de neuroplasticidade cerebral.

A pontuação do MEEM encontrada no presente estudo evidencia um grande comprometimento e déficits cognitivos nos pacientes avaliados. Esses resultados podem estar 
associados com o elevado índice de indivíduos idosos, além da alta demanda de analfabetos associado com as alterações cognitivas provocadas pelo evento vascular.Com os resultados da MIF obteve-se uma maior independência funcional na amostra estudada, corroborando com estudos encontrados na literatura que avaliaram a capacidade funcional de idosos com AVE em Campinas - SP,que encontraram a média da MIF total de 97 sendo resultado sugestivo de independência funcional ${ }^{26}$.

$\mathrm{Na}$ aplicação da escala FM observou-se comprometimento motor grave e resultados semelhantes podem ser observados nos estudos de Cacho, Melo e Oliveira ${ }^{27}$, que avaliaram o comprometimento motor de dez indivíduos hemiplégicos e encontraram para a função motora total da FM $70 \%$ da amostra com comprometimento severo. O comprometimento da função motora proporciona déficits na coordenação dos movimentos, fraqueza muscular, tônus e ajustes posturais anormais e falta de mobilidade, dificultando a realização de atividades de vida diária e reduzindo a qualidade de vida.

Os indivíduos acometidos pelo AVE apresentam alterações de mobilidade, equilíbrio e controle postural, estando mais suscetíveis a eventos de quedas e tendo sua incidência diretamente proporcional ao grau de déficit funcional ${ }^{28}$. No presente estudo, observou-se equilíbrio pobre em $43,59 \%$ dos avaliados com a EEB, um baixo risco de quedas na avaliação da TUG para $52 \%$ da amostra e os resultados da FAC indicam que $46,15 \%$ da amostra apresentam dificuldade parcial ou total e/ou ausência de deambulação. Em um estudo com 30 idosos avaliados, $36 \%$ realizaram o teste do TUG em 30 segundos ou mais, apresentando alto risco de quedas $^{28}$. No estudo com hemiparéticos observouse que dos 21 indivíduos avaliados com a TUG, 19\% levaram mais de 30 segundos para completar o teste, o que indica que os mesmos são considerados dependentes na maioria das AVD e na capacidade de mobilidade ${ }^{29}$. Em um estudo com a aplicação da EEB em 22 casos de AVE, verificouse um risco de quedas em $100 \%$ dos avaliados ${ }^{30}$. A manutenção da postura ereta envolve diversos e constantes ajustes corporais com o propósito de manter os segmentos corporais alinhados e orientados de acordo com a tarefa e as sequelas geradas pelo AVE fazem com que os pacientes empenhem-se na busca do centro de equilíbrio, uma vez que apresentam uma perda sensorial e motora significativa, acarretando maior propensão para eventos de quedas ${ }^{29,30}$. O presente estudo teve como desvantagem um pequeno número de pacientes avaliados. Como limitações, houve extensa dificuldade em rastrear os indivíduos com AVE nos bairros da cidade edetectar dados mais precisos sob a lesão dos pacientes como a localização territorial da lesão e o número de eventos através da comprovação por exames de imagem.

\section{CONCLUSÃO}

O elevado número de acometimento do AVE associado com as características epidemiológicas da região emendam a associação com os déficits funcionais, cognitivos e sensório motores encontrados. Esses achados evidenciam o grave problema de saúde pública que essa patologia representa juntamente com a necessidade da implementação de estratégias preventivas, educacionais e de conscientização da população para reduzir a incidência doAVE. Desse modo,é de grande importância instituir melhorias ou modificações nas estratégias de políticas de saúde em relação ao AVE, como realização de campanhas educativas nas escolas, postos de saúde, hospitais, visando à conscientização e melhoria da qualidade de vida da população. Além disso, torna-se necessário expandir esses conhecimentos através de novos e mais abrangentes estudos científicos para que possa ampliar a margem de conhecimento do AVE.

\section{AGRADECIMENTO}

Reuni - Universidade Federal do Rio Grande do Norte (UFRN); Secretaria Municipal de Saúde de Santa Cruz. 


\section{REFERÊNCIAS}

1. Costa FA, Silva DLA, Rocha VM. Estado neurológico e cognição de pacientes pós-acidente vascular cerebral. Rev Esc Enferm USP. 2011; 45(5):1083-88.

2. Grochovski CR, Camos R, Lima MCAM. Ações de controle dos agravos à saúde em indivíduos acometidos por Acidente Vascular Cerebral. Rev Bras Ci Saude. 2015; 19(4):269-76.

3. Pereira ABNG, Alvarenga $\mathrm{H}$, Pereira Júnior RS, Barbosa MTS. Prevalência de acidente vascular cerebral em idosos no Município de Vassouras, Rio de Janeiro, Brasil, através do rastreamento de dados do Programa Saúde da Família. CadSaúdePública. 2009; 25(9):192936.

4. AppelrosP, Stegmayr B, TerentA. Areview on sex differences in stroke treatment and outcome. Acta NeurolScand. 2010; 121(6):359-69.

5. Ribeiro KSQS, Neves RF, Brito GEG, Morais JD, Lucena EMF, Medeiros JM, Mendes LM. Perfil de usuários acometidos por Acidente Vascular Cerebral adscritos à Estratégia Saúde da Família em uma capital do Nordeste do Brasil. Rev Bras CiSaúde. 2012; 16(2):3544.

6. Datasus: Departamento de informações do SUS. Disponível em: <http://tabnet.datasus.gov.br/tabdata/ cadernos/rn.htm> Acesso em 8/1/2016 ás 22:00.

7. Cavalcante TF, Moreira RP, Araujo TL, Lopes MVO. Fatores demográficos e indicadores de risco de acidente vascular encefálico: Comparação entre moradores do município de Fortaleza e o perfil nacional. Rev Latino-Am Enfermagem. 2010; 18(4):1-6.

8. KellyPJ, Crispino G, Sheehan O, Kelly L, MarnaneM, Merwick A, Hannon N,et al. Incidence, event rates, and early outcome of stroke in Dublin, Ireland: The North Dublin population stroke study. Stroke.2012;43(8):20427.

9. Rosamond W, Flegal K, FurieK, Go A, Greenlund K, Haasen, Ho M,et al. Heart Disease and Stroke Statistics 2007 Update. A Report from the American Heart Association Statistics Committee and Stroke Statistics Subcommittee. Circulation. 2007; 115(5):69-171.

10. Garuzi M, Achitti MCO, Sato CA, Rocha SA, Spagnulo RS. Acolhimento na Estratégia Saúde da Família: Revisão Integrativa. Rev Panam Salud Publica. 2014; 35(2) 144-9.

11. Piassaroli CAP, Aalmeida GC, Luvizotto JC, Suzan ABBM. Modelos de reabilitação fisioterápica em pacientes adultos com sequelas de AVC isquêmico. Rev Neuroc.2012; 20(1):128-137.
12. Teles MS, Gusmão C. Avaliação funcional de pacientes com acidente vascular cerebral utilizando o protocolo de Fugl-Meyer. Rev Neuroc. 2012; 20(1):42-9.

13. Instituto Brasileiro de Geografia e Estatística (IBGE) CIDADES@. Disponível em: <http:// www.cidades.ibge.gov.br/> Acesso em: 18/1/2016.

14. Almeida OP. Mini Exame do Estado Mental e o diagnóstico de demência no Brasil. Arq Neuro-psiquiatr. 1998; 56(3):605-612.

15. Riberto M, Miyazaki MH, Jucá SSH, Sakamoto H, Pinto PPN, Battistella LR. Validação da versão brasileira da Medida de Independência Funcional. Acta Fisiatr. 2004; 11(2):72-76.

16. Berg KO, Maki BE, Williams JI, Holiday PJ, WoodDauphinee SL. Clinical and laboratory measures of postural balance in an elderly population. ArchPhysMedRehabil. 1992; 73(11):1073-80.

17. Fugl-Meyer AR, Jaasko L, Leyman L, Olsson S, Steglind $S$. The poststroke hemiparetic patients: a method for evaluation of physical performance. Scand $\mathrm{J}$ of Rehabil Med. 1975; 7(1):13-31.

18. Mehrholz J, Wagner K, Rutte K, Meisser D, Pohl M. Predictive validity and responsiveness of the Functional Ambulation Category in hemiparetic patients after stroke. Arch Phys Med Rehabil. 2007; 88(10):1314-9.

19. Podsiadlo D, Richardson $S$. The timed up and go: a test of basic mobility for frail persons. Jornal da American Geriatrics Society. 1991; 39(2):142-8.

20. Carvalho JJF, Alvez MB, Viana GA, Machado CB, Santos BF, Kanamura AH, Lottenberg $\mathrm{CL}$, Neto MC,Silva GS.Stroke epidemiology, patterns of management, and outcomes in Fortaleza, Brasil - A hospital-Based Multicenter Prospective Study. Stroke. 2011; 42(12):3341-6.

21. Furukawa TS, Mathias TAF, Marcon SS. Mortalidade por doenças cerebrovasculares por residência e local de ocorrência do óbito: Paraná, Brasil, 2007. Cad Saúde Pública. Rio de Janeiro. 2011; 27(2):327-34.

22. Santos $A B C$, Barreto VP, Oliveira SM, Gomes CAM, Vian KM, Brasil BO, Abrantes TG. Perfil epidemiológico da hipertensão arterial sistêmica na população de Cajazeiras, Paraíba. Rev Bras Ciênc Saúde. 2013; 17(3):253-62.

23. ChavesMLF. Acidente Vascular Encefálico: Conceituação e fatores de risco. Revista Brasileira de Hipertensão. 2000;7(4):332-8.

24. Piassaroli CAP, Almeida GC, Luvizotto JC, Suzan ABBM. Modelos de reabilitação fisioterápica em pacientes adultos com sequelas de AVC isquêmico. Rev Neurocienc 2012; 20(1): 128-137. 
25. Cruz KCT, Diogo MJD. Avaliação da capacidade funcional de idosos com acidente vascular encefálico. Acta Paul Enferm. 2009; 22(5):666-72.

26. Cacho EWA, Melo FRLV, Oliveira R. Avaliação da recuperação motora de pacientes hemiplégicos através do protocolo de desempenho físico Fugl-Meyer. Rev Neurocienc. 2004; 12(2):94-102.

27. Borges PS, Marinho Filho LEN, Mascarenhas $\mathrm{CH}$. Correlação entre equilíbrio e ambiente domiciliar com risco de quedas em idosos com acidente vascular encefálico. Rev Bras Geriatr Gerontol. 2010;13(1):4150.

28. Giriko HC, Azevedo RAN, Kuriki HU, CarvalhoAC. Capacidade funcional de hemiparéticos crônicos submetidos a um programa de fisioterapia em grupo. Fisioterapia e Pesquisa. 2010; 17(3):214-19.
29. Meneghetti $\mathrm{CHZ}$, Delgado GM, Pinto FD, Canonice AP, Gaino MRC. Equilíbrio em indivíduos com acidente vascular encefálico: clínica escola de fisioterapia da Uniararas. Rev Neurocienc. 2009; 17(1):14-18.

\section{Correspondência}

Roberta de Oliveira Cacho

Rua Vila Trairi; s/n; Centro.

CEP 59200-000.

Santa Cruz. Rio Grande do Norte. Brasil.

E-mail: ro_fisio1@hotmail.com 